\title{
Compact fiber-delivered Cr:forsterite laser for nonlinear light microscopy
}

\author{
Ming-Che Chan \\ Tzu-Ming Liu \\ Shih-Peng Tai \\ Chi-Kuang Sun \\ National Taiwan University \\ Department of Electrical Engineering and Graduate \\ Institute of Electro-Optical Engineering \\ Taipei 10617, Taiwan
}

\begin{abstract}
We demonstrate a compact and self-starting fiber-delivered femtosecond Cr:forsterite laser for nonlinear light microscopy. A semiconductor saturable absorber mirror provides the self-starting mechanism and maintains long-term stability in the laser cavity. Four double-chirped mirrors are employed to reduce the size of the cavity and to compensate for group velocity dispersion. Delivered by a large-mode-area photonic crystal fiber, the generated laser pulses can be compressed down to be with a nearly transform-limited pulse width with 2.2-nJ fiber-output pulse energy. Based on this fiberdelivered Cr:forsterite laser source, a compact and reliable twophoton fluorescence microscopy system can thus be realized. $\odot 2005$ Society of Photo-Optical Instrumentation Engineers. [DOI: 10.1117/1.2060586]
\end{abstract}

Keywords: mode-locked Cr:forsterite lasers; double-chirped mirrors; large-modearea photonic crystal fibers; nonlinear light microscopy.

Paper 05011R received Jan. 16, 2005; revised manuscript received Apr. 27, 2005; accepted for publication May 4, 2005; published online Oct. 4, 2005.
Enabled by femtosecond near-infrared (NIR) solid-state light sources, ${ }^{1-6}$ nonlinear light microscopy such as twophoton fluorescence microscopy $(\mathrm{TPFM})^{7}$ and higherharmonic generation microscopy ${ }^{8}$ has attracted much biological and medical interest in recent years due to its capability to provide molecular and structural information with high 3-D spatial resolution. Compared with single-photon excitation, NIR-based nonlinear light microscopy offers the advantages of deeper penetration, higher sample viability, and muchreduced photodamage and photobleaching effects. ${ }^{7-9}$ With their output wavelength covering the infrared penetrating window of most biological tissues at $1200-1300 \mathrm{~nm},{ }^{10}$ femtosecond Cr:forsterite lasers show great promise to serve as excitation sources for nonlinear light microscopy. ${ }^{8,10-14}$ Our recent in vivo studies in live and untreated wild-type zebrafish embryos with a femtosecond $\mathrm{Cr}$ :forsterite laser indicated $>1.5-\mathrm{mm}$ penetration capability while maintaining a submicron resolution $^{12}$ with the third harmonic generation modality. With much-reduced multiphoton absorption in biotissues, ${ }^{13}$ compared with a femtosecond Ti:sapphire laser, no optical damage in live wild-type zebrafish embryos can be observed even with $100-\mathrm{mW}$ incident average power (1-nJ pulse energy) from a femtosecond Cr:forsterite laser continuously illuminating on the embryos after long-term (12-hour) observations, with a total exposure of over $1000 \mathrm{~J}$ applied to one embryo. ${ }^{8}$ Moreover, the near-1300-nm excitation wavelength provided by Cr:forsterite lasers corresponds to the zero dispersion wavelength in most optical fibers, which makes remote-fiber-delivered microscopic systems possible.

For future biomedical or even clinical application, a compact and fiber-delivered $\mathrm{Cr}$ :forsterite laser is desired. Previous studies on Ti:sapphire-based TPFM ${ }^{15}$ have shown that a fiber-

Address all correspondence to Chi-Kuang Sun, National Taiwan University, R319, 2nd EE building, 1 Roosevelt Rd. Sec. 4, Taipei, Taiwan 106 Taiwan. Tel: 886-2-27359703; Fax: 886-2-23677467; E-mail: sun@cc.ee.ntu.edu.tw delivered system is a more compact system arrangement and has better isolation from environmental noises than bulkoptics-based systems. Recently, Thomann and his co-workers have demonstrated a compact and fiber-delivered $\mathrm{Cr}$ :forsterite laser with a $420-\mathrm{MHz}$ repetition rate and $0.43-\mathrm{nJ}$ output pulse energy from optical fibers. ${ }^{16}$ With strong spectral broadening in nonlinear fibers leading to octave-spanning spectra, this previously demonstrated highly repetitive light source is ideal for telecommunication and metrology applications. ${ }^{16}$ For nonlinear light microscopy applications, the fiber-output femtosecond light should provide higher pulse energy $(>1 \mathrm{~nJ})$ and a narrower spectral width $(<15 \mathrm{~nm})$, to achieve higher nonlinear process efficiency while minimizing dispersion effects in biotissues. Since most fluorophores exhibit a lifetime on the order of several nanoseconds, a laser repetition rate on the order of $100 \mathrm{MHz}$, but not much higher, is desired.

In this paper, we report a compact and self-starting fiberdelivered Cr:forsterite laser source designed for nonlinear light microscopy. A semiconductor saturable absorber mirror $(\mathrm{SESAM})^{17}$ is utilized in the laser cavity to maintain longterm stability. Four doubled-chirped mirrors (DCM), ${ }^{18}$ instead of prism pairs, are utilized to reduce the size of the cavity and to compensate for group velocity dispersion dispersion (GVD) arising from the laser crystal. Without any prechirp units, the generated laser pulses are delivered and could be compressed down to be with a nearly transform-limited pulse width by a large-mode-area photonic crystal fiber with 260-mW average output power. With well-controlled selfphase modulation, negligible spectral broadening effect can be achieved. Based on this compact and self-starting femtosecond all-solid-state laser source, a fiber-delivered Cr:forsterite-based TPFM system is demonstrated for the first time.

1083-3668/2005/10(5)/054006/4/\$22.00 @ 2005 SPIE 


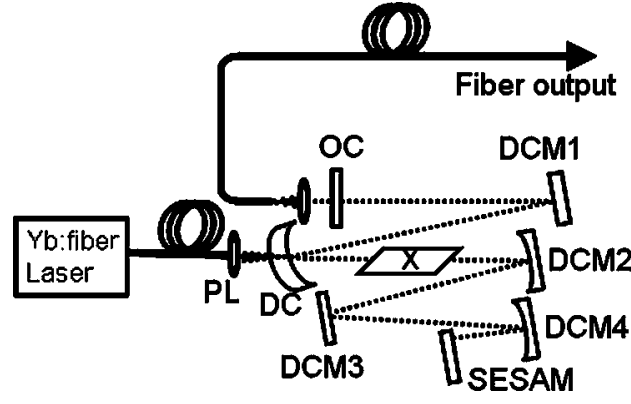

Fig. 1 Schematic diagram of the compact Cr:forsterite laser. PL: pump lens; $D C$ : dichroic curve mirror $(R=10 \mathrm{~cm})$; $D C M 1, D C M 3$ : flat double-chirped mirrors; DCM2, DCM4: curved double-chirped mirrors $(\mathrm{R}=10 \mathrm{~cm})$; SESAM: semiconductor saturable mirror; OC: output coupler; X: Cr:forsterite crystal.

The schematic layout of the compact self-starting fiberdelivered Cr:forsterite laser is shown in Fig. 1. The laser is composed with a single-mode fiber pumping source, a prismless Cr:forsterite laser oscillator, and a single-large-mode photonic crystal fiber for output delivery into a nonlinear light microscope. The pumping source is a cw ytterbium-doped single-mode fiber laser (IPG PYL-10-1064-LP) operating at a 1064-nm wavelength with $10.5-\mathrm{W}$ output power. The pump beam, with a $2.5-\mathrm{mm}$ diameter and 0.35 -mrad divergence, is focused into the Cr:forsterite laser crystal with $\sim 30-\mu \mathrm{m}$ beam radius through a pump lens with a $10-\mathrm{cm}$ focal distance. The laser oscillator is composed of one dichroic curved mirror (DC), a Cr:forsterite crystal, a SESAM, two plane DCMs (DCM1, DCM3), two curved DCMs (DCM2, DCM4), and an output coupler (OC). We use a standard z-fold cavity for astigmatism compensation. The repetition rate of the laser oscillator was designed to be $120 \mathrm{MHz}$ to maintain an output pulse energy well above $1 \mathrm{~nJ}$ for nonlinear light microscopy. Higher repetition rate is possible for other applications. ${ }^{19}$ The radius of curvatures of the focusing mirrors (DC, DCM2, and DCM4) are $10 \mathrm{~cm}$. DC and DCM2 are separated with $10.6 \mathrm{~cm}$ and the lengths of both $\mathrm{z}$-folded cavity arms are $62 \mathrm{~cm}$, which result in a 1.24-m total cavity length. The $\mathrm{Cr}-$ :forsterite crystal is a 5- $\times 5-\times 11.4-\mathrm{mm}$ Brewster-cut crystal with an absorption coefficient of $1.5 \mathrm{~cm}^{-1}$. Both the orientations of the pump and the oscillator electric fields are parallel to the $\mathrm{b}$ axis of the $\mathrm{Cr}$ :forsterite crystal. The crystal is cooled down to $-2{ }^{\circ} \mathrm{C}$ by a liquid and a TE cooler. The surface of the crystal is purged with dry nitrogen to prevent water condensation. The SESAM with a picosecond transient time is for self-starting and enhancement of the mode-locking force. It consists of 25 periods of a GaAs/AlAs Bragg reflector, followed by an $\mathrm{Al}_{0.48} \mathrm{In}_{0.52}$ As quarter-wave layer with two embedded $\mathrm{Ga}_{0.47} \mathrm{In}_{0.53}$ As quantum wells. To provide the saturable absorber nonlinearity for initiating and stabilizing the Cr:forsterite laser, the quantum-well structure was designed to have the heavy-hole excitonic resonance around $1230 \mathrm{~nm}$ at room temperature. The insertion loss of the SESAM is $2.5 \%$ with a saturation fluence of $\sim 50 \mu \mathrm{J} / \mathrm{cm}^{2}$. The laser beam is focused with a curved mirror (DCM4) onto the SESAM. The beam radius of the cavity transverse mode on the SESAM is about $30 \mu \mathrm{m}$. The double-pass group velocity dispersion (GVD) of the laser crystal was estimated to be $\sim 568 \mathrm{fs}^{2}$
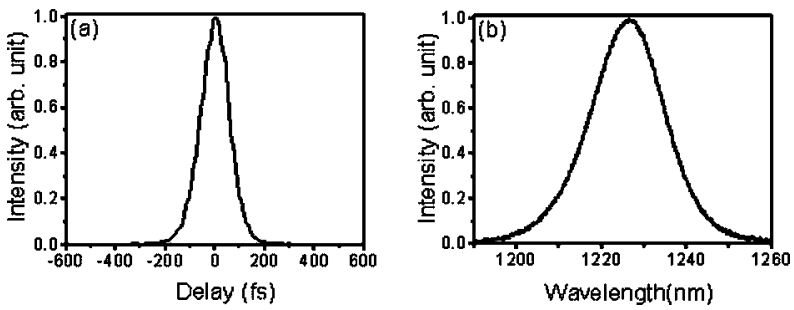

Fig. 2 (a) Autocorrelation traces and (b) spectra generated by laser with $2 \%$ output coupler. Laser output power: $100 \mathrm{~mW}$. Laser repetition rate: $100 \mathrm{MHz}$. Net cavity dispersion: $-332 \mathrm{fs}^{2}$. Laser pulse width: 85 fs. Full-width-half-maximum spectral width: $20 \mathrm{~nm}$.

around $1230 \mathrm{~nm}^{20}$ To compensate for positive GVD arising from the laser crystal, four DCMs ${ }^{18}$ are adopted in the laser cavity for a compact cavity design. On the surface of each DCM, the period of dielectric coating is chirped, which results in the required negative dispersion. An impedance matching section and antireflection coatings were grown above the chirped region to avoid group delay oscillation and resulted in broadband mirrors with a controlled dispersion, which are important for the further development of a tunable ultrashort pulse laser. ${ }^{18}$ In our experiment, each doublechirped mirror was designed with a wavelength from 1200 to $1400 \mathrm{~nm}$ and provided $-150-\mathrm{fs}^{2}$ GVD around $1230 \mathrm{~nm} .{ }^{20}$ By folding the cavity with DCMs, we cannot only reduce the size of the cavity but also accomplish a net cavity GVD of $-632 \mathrm{fs}^{2}$ at $1230 \mathrm{~nm}$ for stable mode locking. Due to the prismless cavity design, the optical part of the laser system can be packaged within a box with $20-\times 30-\times 20-\mathrm{cm}^{3}$ dimension, which is on the order of or smaller than the size of a scanning microscope.

The performance of the Cr:forsterite oscillator can be tuned by changing output couplers with different ratios, by changing intracavity dispersion by replacing DCM3 with a high-reflection mirror, and by fine-tuning cavity length and positions of DCM2 or SESAM with different working points. To generate stable mode-locked pulses, the net cavity dispersion should be in a slightly negative dispersion region. With two double-chirped mirrors (four bounces), the net cavity dispersion is $-24 \mathrm{fs}^{2}$ and the mode-locking behavior is not stable. With three double-chirped mirrors (six bounces), the net cavity dispersion is $-324 \mathrm{fs}^{2}$, which is suitable for generating transform-limited pulses. However, in SESAM-assisted Kerr-lens mode-locking mechanism, too much intracavity dispersion will result in multiple-pulsing effects, which set an upper limit for output power. To increase the upper limit, the net cavity dispersion should be more negative. Thus, four double-chirped mirrors (eight bounces) were utilized in our laser cavity. However, applying even more double-chirped mirrors will increase the size of the laser cavity, which is unsuitable for clinical nonlinear light microscopy.

At 10.5 -W pump power, with a $2 \%$ to $10 \%$ output coupler, the laser oscillator can emit femtosecond pulses with 100 $\sim 530$ - $\mathrm{mW}$ output power, 10 20-nm full-width-halfmaximum (FWHM) bandwidth, and 85 319-fs pulse duration (see Figs. 2 and 3). The threshold pump power for $2 \%$ and $10 \%$ output couplers are $3.8 \mathrm{~W}$ and $4.6 \mathrm{~W}$, respectively. For nonlinear light microscopy applications, we selected a 

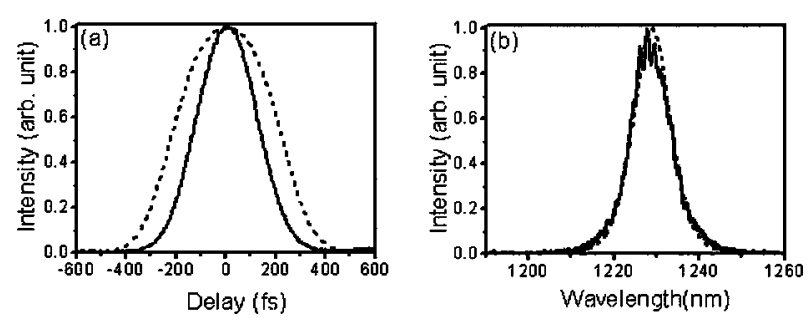

Fig. 3 (a) Autocorrelation traces and (b) spectra generated by laser with $10 \%$ output coupler (dotted lines) and delivered through a largemode-area photonic crystal fiber (solid lines). Laser output power: $530 \mathrm{~mW}$. Laser repetition rate: $120 \mathrm{MHz}$. Net cavity dispersion: $-632 \mathrm{fs}^{2}$. Laser pulsewidth: $320 \mathrm{fs}$. Full-width-half-maximum spectral width: $10.2 \mathrm{~nm}$. Fiber-output power: $260 \mathrm{~mW}$. Fiber output pulse width: 204 fs. Fiber output full-width-half-maximum spectral width: $10.4 \mathrm{~nm}$.

$10 \%$ output coupler to maximize the generated laser pulse energy. When propagating through optical fibers, transformedlimited femtosecond pulses broaden quickly due to selfphase-modulation effect and dispersion effect inside optical fibers. To minimize the self-phase-modulation effect and to pre-compensate the dispersion effect in the delivery fiber, we operate the laser with a smaller FWHM bandwidth with a positively chirped output pulse. The dotted lines in Fig. 3(a) and Fig. 3(b) show the measured autocorrelation traces and spectrum of the mode-locked laser output before entering into the PCF. The FWHM of laser spectrum was $10.2 \mathrm{~nm}$ with a 1229-nm central wavelength. The measured pulse duration was 319 fs assuming a Gaussian pulse shape. With a strong chirp, the time-bandwidth product of the laser output was 0.65 .

To isolate environment disturbance and for convenient and reliable light delivery, fiber-coupled systems have been widely adopted in all commercial cw laser-based confocal scanning microscopes. Thus, in nonlinear light microscopy for clinical applications, due to the issues of convenience and reliability, developing a fiber-coupled nonlinear light microscope similar to a commercial confocal one is necessary. However, for femtosecond Ti:sapphire-laser-based TPFM, previous studies on single-mode fiber delivery indicated strong image intensity degradation due to pulse lengthening, mainly contributed from dispersion and nonlinear self-phase modulation effects. ${ }^{15}$ Although the spectrum generated by $\mathrm{Cr}$ :forsterite lasers is located near dispersion zero wavelength of fibers, strong self-phase modulation was still observed in a previous work even with $0.43-n J$ pulse energy. ${ }^{16}$ With more than 1-nJ pulse energy from our constructed compact laser, the self-phase modulation induced pulse-duration degradation will be worse. Suitable delivery fiber instead of traditional single-mode fiber should be selected. In recent years, with the advances of PCFs, several groups have demonstrated delivery of nanojoule femtosecond pulses from Ti:sapphire lasers through a large-mode area $\mathrm{PCF}^{21}$ and a hollow-core $\mathrm{PCF}^{22,23}$ Self-phase-modulation induced pulse broadening was diminished inside these fibers. However, prechirping units were still required for dispersion compensation ${ }^{21}$ of large-core PCFs. In this work, without prechirping units, delivery of femtosecond Cr:forsterite laser pulses through a large-mode area PCF is demonstrated.
We delivered the generated $\sim 1230$-nm femtosecond pulses with a 65-cm-long large-mode area PCF (LMA 35, Crystal-Fiber A/S). We choose the large-mode area PCF to output laser pulses not only due to its large effective mode area with diminished nonlinear effects but also due to its slight negative dispersion at $1230 \mathrm{~nm}$ so that the chirping of the laser pulses could be compensated during fiber delivery. The incident laser beams were linearly polarized. The polarizations didn't change during fiber delivery. The LMA-35 PCF is single mode at the 1230-nm wavelength with a modefield area of $530 \mu \mathrm{m}^{2}$. The dispersion and its slope at $1550 \mathrm{~nm}$ are $25 \mathrm{ps} / \mathrm{nm} / \mathrm{km}$ and $0.07 \mathrm{ps} / \mathrm{nm}^{2} / \mathrm{km}$, respectively. ${ }^{24}$ The solid lines in Fig. 3(a) and Fig. 3(b) show the autocorrelation trace and spectrum after optical pulse passing through the PCF. With 260-mW output power, corresponding to an output pulse energy of $2.2 \mathrm{~nJ}$, no significant spectral broadening effect due to self-phase modulation can be observed, in contrast to the previous nonlinear fiber experiment. ${ }^{16}$ On the other hand, with negative dispersion compensation provided by the PCF, the width of the chirped pulses from the laser oscillator was successfully compressed down to 200 fs [straight line in Fig. 3(a), assuming a Gaussian pulse shape] with a time-bandwidth product of 0.44 , which is transform-limited. The pulse durations and FWHM spectral widths of the delivered pulses were found to be independent of the polarizations of the input laser beams. From an independent calculation, we can also estimate the maximum nonlinear phase shift experienced by the optical pulses inside the PCF to be on the order or less than $0.5 \pi$. With a narrow spectral width, a short pulse width, 2-nJ pulse energy, and a central wavelength located at the biopenetration window, this self-started compact $\mathrm{Cr}$ :forsterite laser is an ideal light source for nonlinear light microscopy.

We set up a fiber-delivered two-photon-fluorescence microscope (TPFM) system based on the demonstrated Cr:forsterite laser. The PCF fiber is connected to the input end of an Olympus FV300 scanning system combined with an Olympus BX51 upright microscope while all optics were modified to allow the passage of $1200 \sim 1350-\mathrm{nm}$ infrared (IR) light. The biological sample used to study imaging performance of this PCF-fiber based TPFM is the leaf of Rhaphidophora aurea, and a water immersion objective (Olympus, LUMPlanFI/IR, $0.9 \mathrm{NA}$ ) is used to focus the laser beam into the sample. To guarantee the collected signals, two-photon fluorescence of chlorophyll, a short wave pass color filter (Lambda Research Optics SWP-1202U-700), and a 40-nm bandwidth interference filter (Lambda Optics 670-F40-12.7), corresponding to the $670-\mathrm{nm}$ two-photon fluorescence wavelength of chlorophyll, ${ }^{10}$ are inserted before the photomultiplication tube in the Olympus FV300 scanning unit. The acquired twophoton fluorescence (chlorophyll) images of the mesophyll tissues in a fresh leaf of Rhaphidophora aurea are shown in Fig. 4. The average illumination power on the leaf is decayed down to $5 \mathrm{~mW}$. From Fig. 4, chloroplasts distribution inside the mesophyll cells can be identified with a submicron spatial resolution. This result demonstrates that this fiber-delivered compact $\mathrm{Cr}$ :forsterite laser is an ideal source for nonlinear light microscopy. To the best of our knowledge, this is the first fiber-based nonlinear microscope where the fiber can actually increase the excitation efficiency of nonlinear signals through 


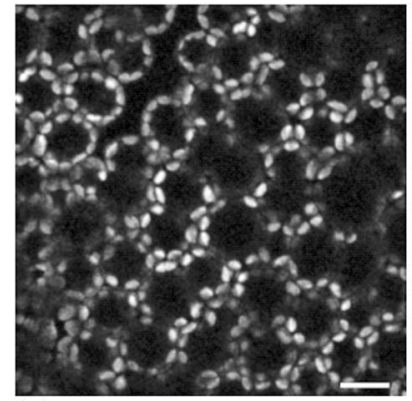

(a)

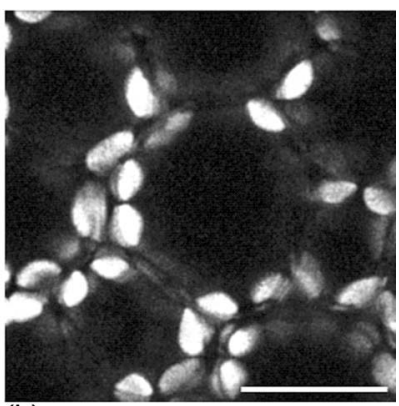

(b)

Fig. 4 Two-photon fluorescence image of mesophyll tissue in the leaf of Rhaphidophora aurea, taken with the system based on a large-core photonic crystal fiber with a size of $240 \times 240 \mu \mathrm{m}$ (a) and 80 $\times 80 \mu \mathrm{m}$ (b). Scale bar: $30 \mu \mathrm{m}$.

compressing the optical pulse width without the help of a dispersion compensation unit.

In conclusion, we have successfully demonstrated a compact, prismless, self-starting, and fiber-delivered femtosecond $\mathrm{Cr}$ :forsterite laser for nonlinear light microscopy by employing double-chirped mirrors and a SESAM. Delivered by a large-mode-area photonic crystal fiber, the generated laser pulses can be compressed down to be within a nearly transform-limited pulse width with 2.2-nJ fiber-output pulse energy. Based on this fiber-delivered Cr:forsterite laser source, a compact and reliable two-photon fluorescence microscopy system can thus be realized.

\section{Acknowledgments}

The authors are indebted to Professor Franz X. Kärtner and James G. Fujimoto at Massachusetts Institute of Technology for providing the double-chirped mirrors. The authors also gratefully acknowledge financial support under the National Health Research Institute (NHRI-EX93-9201EI) and the National Taiwan University Center for Genomic Medicine.

\section{References}

1. J. M. Evans, V. Petricevic, A. B. Bykov, A. Delgado, and R. R. Alfano, "Direct diode-pumped continuous-wave near-infrared tunable laser operation of $\mathrm{Cr}^{4+}$ forsterite and $\mathrm{Cr}^{4+} \mathrm{Ca}_{2} \mathrm{GeO}_{4}$, " Opt. Lett. 22, 1171-1173 (1997).

2. A. Robertson, H. Fuchs, U. Ernst, R. Wallenstein, V. Scheuer, and T. Tschudi, "Prismless femtosecond Cr:forsterite laser," J. Opt. Soc. Am. B 17, 668-671 (2000).

3. C. Chudoba, J. G. Fujimoto, E. P. Ippen, H. A. Haus, U. Morgner, F. X. Kärtner, V. Scheuer, G. Angelow, and T. Tschudi, "All-solid-state Crforsterite laser generating 14-fs pulses at $1.3 \mu \mathrm{m}$," Opt. Lett. 26, 292-294 (2001).

4. A. Sennaroglu, "Broadly tunable $\mathrm{Cr}^{4+}$-doped solid-state lasers in the near infrared and visible," Prog. Quantum Electron. 26, 287-352 (2002).

5. A. Agnesi, A. Guandalini, A. Lucca, G. Reali, A. Tomaselli, and C. Vacchi, "Low misalignment sensitivity Kerr-lens mode-locked femtosecond $\mathrm{Cr}^{4+}$ :Forsterite laser for nonlinear microscopy," IEEE J.
Quantum Electron. 40, 1569-1574 (2004).

6. Y. Kalisky, " $\mathrm{Cr}^{4+}$-doped crystals: their use as lasers and passive Q-switches," Prog. Quantum Electron. 28, 249-303 (2004).

7. W. Denk, J. H. Strickler, and W. W. Webb, "2-Photon laser scanning fluorescence microscopy," Science 248, 73-76 (1990).

8. C.-K. Sun, S.-W. Chu, S.-Y. Chen, T.-H. Tsai, T.-M. Liu, C.-Y. Lin, and H.-J. Tsai, "Higher harmonic generation microscopy for developmental biology," J. Struct. Biol. 147, 19-30 (2004).

9. J. M. Squirrell, D. L. Wokosin, J. G. White, and B. D. Bavister, "Long-term two-photon fluorescence imaging of mammalian embryos without compromising viability," Nat. Biotechnol. 17, 763-767 (1999).

10. S.-W. Chu, I.-H. Chen, T.-M. Liu, P. C. Chen, C.-K. Sun, and B.-L. Lin, "Multimodal nonlinear spectral microscopy based on a femtosecond Cr:forsterite laser," Opt. Lett. 26, 1909-1911 (2001).

11. T.-M. Liu, S.-W. Chu, C.-K. Sun, B.-L. Lin, P.-C. Cheng, and I. Johnson, "Multiphoton confocal microscopy using a femtosecond Cr:Forsterite laser," Scanning 23, 249-254 (2001).

12. S.-W. Chu, S.-Y. Chen, T.-H. Tsai, T.-M. Liu, C.-Y. Lin, H.-J. Tsai, and C.-K. Sun, "In vivo developmental biology study using noninvasive multi-harmonic generation microscopy," Opt. Express 11, 3093 3099 (2003).

13. I.-H. Chen, S.-W. Chu, C.-K. Sun, P. C. Cheng, and B.-L. Lin, "Wavelength dependent damage in biological multi-photon confocal microscopy: A micro-spectroscopic comparison between femtosecond Ti:sapphire and Cr:forsterite laser sources," Opt. Quantum Electron. 34, 1251-1266 (2002).

14. C.-K. Sun, C.-C. Chen, S.-W. Chu, T.-H. Tsai, Y.-C. Chen, and B.-L. Lin, "Multiharmonic generation biopsy of skin," Opt. Lett. 28, 24882490 (2003).

15. D. Bird and M. Gu, "Compact two-photon fluorescence microscope based on a single-mode fiber coupler," Opt. Lett. 27, 1031-1033 (2002).

16. I. Thomann, A. Bartels, K. L. Corwin, N. R. Newbury, L. Hollberg, S. A. Diddams, J. W. Nicholson, and M. F. Yan, "420-MHz Cr:forsterite femtosecond ring laser and continuum generation in the 1 $\sim 2$ - $\mu \mathrm{m}$ range," Opt. Lett. 28, 1368-1370 (2003).

17. U. Keller, D. A. B. Miller, G. D. Boyd, T. H. Chiu, J. F. Ferguson, and M. T. Asom, "Solid-state low-loss intracavity saturable absorber for Nd:YLF lasers: an antiresonant semiconductor Fabry-Perot saturable absorber," Opt. Lett. 17, 505-507 (1992).

18. N. Matuschek, F. X. Kärtner, and U. Keller, "Theory of doublechirped mirrors," IEEE J. Sel. Top. Quantum Electron. 4, 197-208 (1998).

19. T.-M. Liu, F. X. Kärtner, J. G. Fujimoto, and C.-K. Sun, "Multiplying the repetition rate of passive mode-locked femtosecond lasers by an intracavity flat surface with low reflectivity," Opt. Lett. 30, 439-441 (2005).

20. I. Thomann, L. Hollberg, S. A. Diddams, and R. Equall, "Chromiumdoped forsterite: dispersion measurement with white-light interferometry," Appl. Opt. 42, 1661-1666 (2003).

21. D. G. Ouzounov, K. D. Moll, M. A. Forster, W. R. Zipfel, W. W. Web, and A. L. Gaeta, "Delivery of nanojoule femtosecond pulses through large-core microstructure fibers," Opt. Lett. 27, 1513-1515 (2002).

22. W. Göbel, A. Nimmerjahn, and F. Helmchen, "Distortion-free delivery of nanojoule femtosecond pulses from a Ti:sapphire laser through a hollow-core photonic crystal fiber," Opt. Lett. 29, 1285-1287 (2004).

23. S.-P. Tai, M.-C. Chan, T.-H. Tsai, S.-H. Guol, L.-J. Chen, and C.-K. Sun, "Two-photon fluorescence microscope with a hollow-core photonic crystal fiber," Opt. Express 12, 6122-6128 (2004).

24. From the published data at http://www.crystal-fibre.com/Products/ large_mode_area/datasheets/LMA-35.pdf/, we can estimate the dispersion zero wavelength to be $\sim 1190 \mathrm{~nm}$. The negative dispersion at $1230 \mathrm{~nm}$ is $\sim 2.6 \mathrm{ps} / \mathrm{nm} / \mathrm{km}$. 